\title{
Proliferation, steroid receptors and clinical/pathological response in breast cancer treated with letrozole
}

\author{
WR Miller",', S White', JM Dixon', J Murray', L Renshaw' and TJ Anderson' \\ 'The Edinburgh Breast Unit, Western General Hospital, Edinburgh EH4 2XU, UK
}

\begin{abstract}
Sixty-three postmenopausal women with large primary breast cancers were treated with neoadjuvant letrozole ( $2.5 \mathrm{mg}$ daily) for 3 months. Tumour samples were taken at diagnosis and after 10- 14 days and 3 months treatment. Immunohistochemical staining for Ki67, oestrogen receptor (ER) and progesterone receptor (PgR) was performed and related to clinical (ClinR) and pathological responses (PathR) after 3 months treatment. ClinR was observed in 48 of 63 cases (76.2\%) and PathR in 47 of 62 (75.8\%). Pretreatment Ki67 scores were similar in responders (R) and non-responders (NR). Highly significant Ki67 decreases occurred in all tumour subgroups at $10-14$ days $(P<0.005)$. A significant difference in Ki67 scores at $10-14$ days $(P<0.007)$ was found between PathR and PathNR but not between ClinR and ClinNR. At 3 months, decreases from pretreatment Ki67 scores were highly significant in all tumour subgroups irrespective of response status. However, whereas Ki67 scores were significantly different between pathological $R$ and NR $(P=0.009)$, the corresponding comparison of ClinR status was not. Significant decreases between 10 - I 4 days and 3 months were found only in ClinR and PathR $(P=0.02$ and 0.045 , respectively). Treatment significantly reduced $P g R$ expression at 14 days and 3 months (both $P<0.000$ I), but the level of changes was not different between response status groups. In summary, letrozole produces rapid and profound decreases in expression of Ki67 and PgR but changes do not always correlate with clinical and pathological responses.

British Journal of Cancer (2006) 94, I05 I- 1056. doi: I0.1038/sj.bjc.660300 I www.bjcancer.com
\end{abstract}

Published online 14 March 2006

(c) 2006 Cancer Research UK

Keywords: breast cancer; neoadjuvant therapy; aromatase inhibitor; proliferation; steroid receptors; clinical response

Letrozole is a third-generation aromatase inhibitor, which in clinical trials has been shown to be highly effective in postmenopausal women with oestrogen receptor (ER)-positive breast cancer (Eiermann et al, 2001; Mouridsen et al, 2001; Goss et al, 2003; Rose et al, 2003). Previous studies using neoadjuvant therapy have shown that letrozole may produce profound changes in tumour pathology and immunohistochemical markers (Dixon et al, 2001; Ellis et al, 2001, 2003; Miller et al, 2003; Anderson et al, 2004). Furthermore, it is clear that the clinical effects of neoadjuvant treatment with third-generation aromatase inhibitors in postmenopausal women are not dissimilar to those seen with neoadjuvant chemotherapy (Dixon et al, 2001) and are achieved with less morbidity. Comparative studies have also shown that the effects of third-generation aromatase inhibitors are more consistent and greater than tamoxifen on proliferation (as measured by Ki67) and markers of oestrogen action (progesterone receptors (PgR) and trefoil factor 1) (Ellis et al, 2003; Miller et al, 2003; Anderson et al, 2004). It is therefore interesting that in the neoadjuvant setting, letrozole yields significantly superior clinical results than tamoxifen and also appears to be more effective in particular subgroups such as tumours with low ER levels and overexpression of HER-2 (Ellis et al, 2001). However, the timescale of these effects and their relationship to clinical and pathological

*Correspondence: Dr WR Miller; E-mail: w.r.miller@ed.ac.uk Received II August 2005; revised 3 January 2006; accepted 17 January 2006; published online 14 March 2006 response as assessed at the end of treatment has yet to be fully defined. The aim of the present study was therefore to assess the effects of letrozole on the proliferation marker Ki67 and receptors for oestrogen and progesterone by immunohistochemical assessment in serial biopsies from primary breast cancers taken before, at $10-14$ days and at 3 months into treatment.

\section{MATERIALS AND METHODS}

\section{Patients}

A total of 63 postmenopausal women presented to the Edinburgh Breast Unit with large $(>3 \mathrm{~cm})$ primary breast cancer, which were ER-rich (Allred score 5-8). (However, review of the cases in the research laboratory showed that all patients recruited to the study had ER scores of 7 or 8.) All patients apart from 12 were technically operable. The primary clinical objective was to downstage tumours such that those who were inoperable became amenable for surgery and those who would have required mastectomy could become candidates for breast conservation. This series represents consecutive patients recruited but excluding cases in which the tumour was shown to be multifocal or of special histological type (e.g. mucinous, tubular/cribriform and lobular). All patients gave informed consent to be included in the study, which had been approved by the local ethics committee (2001/W/BU/09 and 2001/ $\mathrm{W} / \mathrm{BU} / 10)$ 


\section{Treatment}

All patients received letrozole $(2.5 \mathrm{mg}$, daily) for 3 months. Serial measurements of the primary tumours were taken before, at 6 weeks and at 3 months by calliper and ultrasound as described previously (Forouhi et al, 1994; Dixon, 2001). The tumour was also imaged mammographically before and at 3 months. Core biopsies were taken at the start, after 10-14 days and at 3 months of treatment as described previously (Iqbal et al, 2002). All patients, apart from eight patients (who electively continued on treatment), received definitive surgery at 3 months.

\section{Response assessment}

Tumour volumes were determined from ulstrasound measurements as described by Forouhi et al (1994). Reduction in volume over a 3 month period $>50 \%$ was regarded as clinical response; this includes both complete and partial responders.

Pathological response was determined by comparing biopsies taken before and after 3 months of treatment. Only marked reduction in cellularity and/or a clear increase in fibrosis were used as evidence of pathological response. Although these changes are essentially subjective, they were confirmed by two observers working independently. The criteria may underestimate actual morphological changes occurring to a lesser degree, but these were excluded because of the difficulty in comparing histological appearances on limited tissue such as in core biopsies with those in the more substantial material obtained at tumour excision. No case was classified as a complete PathR, residual evidence of malignant cells being evident.

\section{Immunohistochemistry}

Immunohistochemistry staining with antibody to MIB1 (Ki-67) antigen (Europath Ltd, Cornwall, UK) diluted $\times 50$ was used as a measure of tumour cell proliferation. Reactivity was detected by an $\mathrm{ABC}$ - peroxidase-antiperoxidase (PAP) method, and scored according to the method described by Going (1994). A change of $>40 \%$ between different paired biopsies was taken as being meaningful (Ellis et al, 1998; Iqbal et al, 2002) and a value of $<1 \%$ was regarded as indicating a lack of proliferation.

Reactivity for ER or PgR was performed by the PAP method, after microwave antigen retrieval, using $\mathrm{ER} \alpha$ antibody clone $6 \mathrm{f} 11 / 2$ (Novocastra Ltd, Newcastle, UK) and PgR antibody clone PgR636 (DAKO Labs, Ely, UK) using the DAKO EnVision system according to the manufacturer's instruction. Results were scored on a scale of $0-3$ for staining intensity (with each successive score denoting increasing intensity), and on a score of $0-5$ for increasing proportion of positive cancer nuclei $(0=$ none, $1=<1 \%, \quad 2=1-10 \%, 3=11-33 \%, \quad 4=34-66 \%, \quad 5=>66 \%)$. The values were then summed into a category score within a range of 0-8 (Allred et al, 1998).

\section{Statistics}

Non-parametric comparisons using either Wilcoxon rank or Spearman paired testing was employed and, where appropriate, $3 \times 2 \chi^{2}$ testing.

\section{RESULTS}

\section{Clinical and pathological response}

Of the 63 patients, $48(76.2 \%)$ were classified as clinical responders (ClinR). With regard to pathological response, one case was not assessable because of insufficient material after biopsy at 3 months. Of the remaining 62 cases, $47(75.8 \%)$ had clear evidence of pathological response. Although response rates were similar, there was not exact concordance between clinical and pathological outcomes. Thus, 42 of $48 \mathrm{ClinR}$ were also pathological responders (PathR) (six were pathological non-responders (PathNR)) and nine of 15 clinical non-responders (ClinNR) were also PathNR. Of the remaining six cases, five were PathR and one case was pathologically not assessable.

\section{Proliferation (Ki67)}

The tumour Ki67 scores for pretreatment, 10-14 days and 3 months samples, subdivided according to clinical and pathological response status, are shown in Table 1.

No significant differences were apparent for pretreatment group scores between responders (R) and non-responders (NR) whether assessed clinically or pathologically. However, at 10-14 days, whereas there was no difference between ClinR and ClinNR, the PathNR were significantly higher than PathR $(P=0.024)$. Similarly at 3 months, although there was no significant difference between ClinR and ClinNR, values in PathNR were significantly higher than in PathR $(P=0.009)$.

With regard to group changes in Ki67 with treatment, the scores at 10-14 days and 3 months were highly significantly decreased, as compared with those in paired pretreatment biopsies. These changes were irrespective of clinical or pathological response status. In terms of changes occurring between 10-14 days and 3 months, a significant decrease was seen in ClinR $(P=0.02)$ and PathR $(P=0.045)$. However, values were not significantly different between 10-14 days and 3 months in ClinNR and PathNR.

Changes in Ki67 with treatment in individual tumours are summarised in Table 2 . In terms of $>40 \%$ change criteria for ClinR, 52 of 63 cases $(82.5 \%)$ showed a decrease at $10-14$ days, and 53 of 63 tumours (84\%) showed a reduction at 3 months. These reductions were seen in both ClinR and ClinNR groups, and there was no significant difference between them. However, for pathological assessments, there was a significant difference between PathR and PathNR at 10-14 days $(P=0.034)$ but not at 3 months. Treatment effects were also assessed on the basis of reducing Ki67 scores to $<1 \%$. It can be seen that whereas 11 cases $(17.5 \%)$ were reduced to $<1 \%$ by $10-14$ days, these numbers markedly increased to $27(42.9 \%)$ by 3 months. Whereas the reduction to $<1 \%$ was seen in both responding and nonresponding tumours, the increase in numbers between 10-14 days and 3 months is predominantly associated with responding tumours.

Different patterns of changes in Ki67 over the treatment period could be detected. These are shown in Figure 1. The largest cohort of tumours (47) showed substantial decreases at 10-14 days, which were maintained or fell further at 3 months (in these tumours, changes in Ki67 at 10-14 days were predictive of those at 3 months). However, there was a small cohort of five patients in which a decrease at 10-14 days was followed by a substantial rise

Table I Ki67 scores in tumour taken before and after 10-14 days and 3 months of treatment, subdivided according to response status

Ki67 scores (mean \pm s.e.m.)

Pretreatment 10-14 days 3 months

Clinical responders (48) $\quad 14.17 \pm 1.10 \quad 5.11 \pm 0.98^{+} 4.13 \pm 0.96^{+} \bullet$

Clinical non-responders (15) $\quad 15.29+2.06 \quad 6.72+2.04^{+} \quad 5.85+\left.1.9\right|^{* * 0}$

$P=0.70 \quad P=0.77 \quad P=0.13$

$14.03+1.09 \quad 4.02+085^{+} \quad 3.47+0.90^{+} \bullet$

Pathological responders (47)

Pathological non-responders (I5) $\quad 15.97 \pm 2.22 \quad 9.35 \pm 2.22 * * 8.11 \pm 2.01 * * *, \circ$

$$
P=0.52 \quad P=0.024 \quad P=0.009
$$

Compared with pretreatment tumour: ${ }^{+} P<0.000$ I; $* P=0.003 ; \quad * * P=0.007$ *** $P=0.009$. Compared with tumour taken after 10-14 days of treatment: $\bullet P=0.02 ; \bullet P=0.045:{ }^{\circ} P=N S$. 


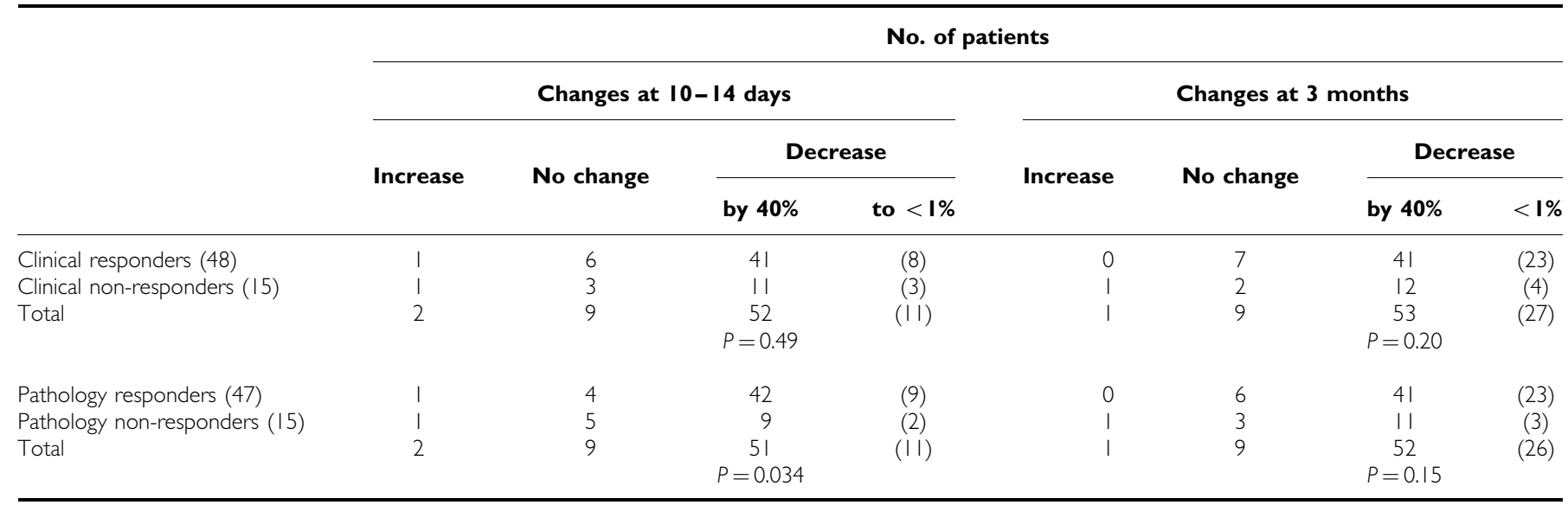

Statistical comparison of increase $(>40 \%)$, decrease $(>40 \%)$ or no change $(<40 \%)$ compared with pretreatment values by $3 \times 2$ chi-square testing.
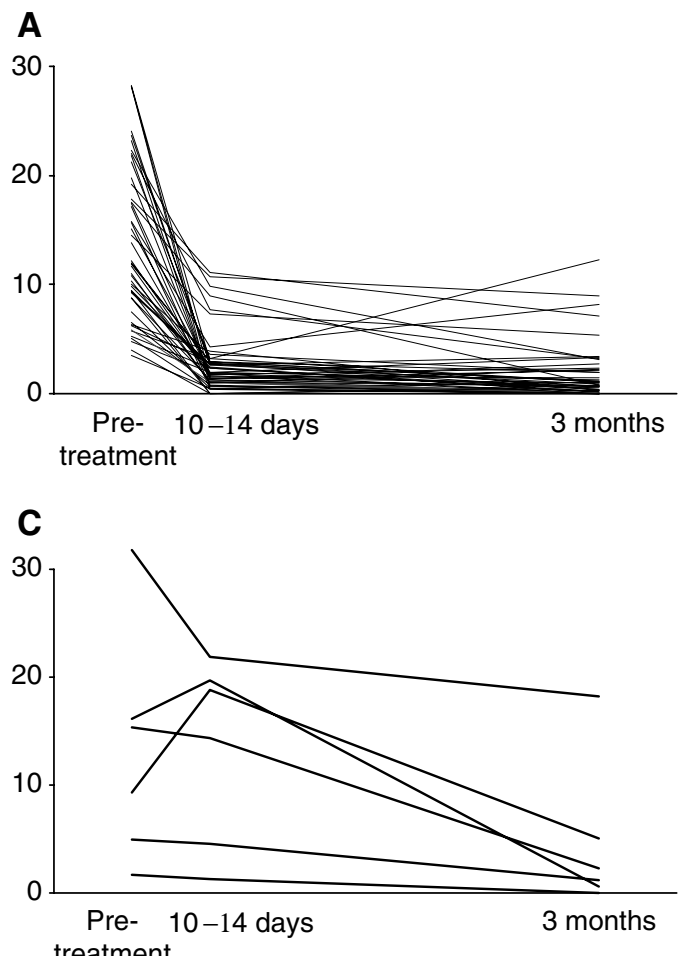

B

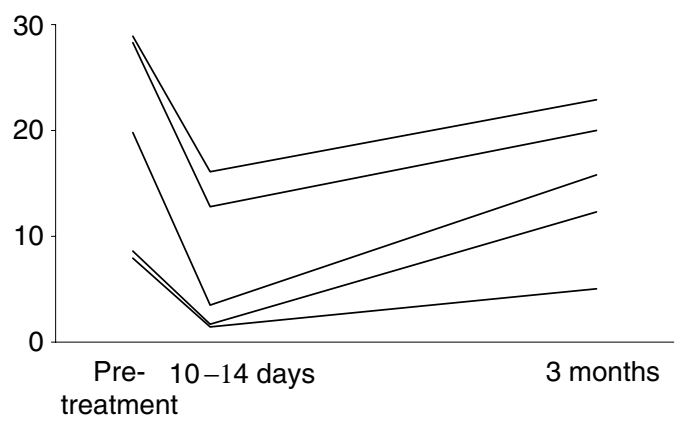

D

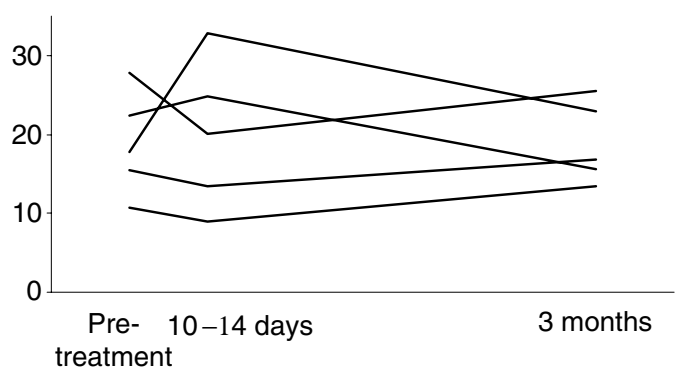

Figure I Tumour Ki67 scores before and after 10 - 14 days and 3 months treatment with letrozole. (A) Cases that show decreases ( $>40 \%$ ) at both $10-$ 14 days and 3 months. (B) Cases that show decreases (>40\%) at 10-14 days but not at 3 months. (C) Cases that show no change at $10-14$ days but a decrease (>40\%) at 3 months. (D) Cases that show no decrease at either 10-14 days or 3 months.

in score at 3 months (in these tumours, changes in Ki67 at 10-14 days did not therefore concur with those at 3 months). Of the 11 that did not decrease at 10-14 days, six decreased at 3 months (Ki67 changes therefore did not concur at 10-14 days and 3 months) and five were not reduced at 3 months (lack of change in Ki67 at 10-14 days was predictive of no change at 3 months). These patterns of Ki67 did not correlate with ClinR and PathR phenotype (Figure 2).

\section{Progesterone receptors}

Of the 63 cases, 57 (90\%) were $\mathrm{PgR}+$ ve; of the six negatives, five were ClinR and four were PathR. The changes with treatment of
PgR staining subdivided according to ClinR and PathR status are summarised in Table 3.

Treatment reduced PgR scores such that, at 10-14 days, values were significantly lower than those in the paired pretreatment biopsy $(P<0.0001)$. This decrease was found in all tumour subgroups irrespective of clinical or pathological assessment status. Similar highly statistically significant decreases were also seen when pretreatment values were compared with corresponding pairs at 3 months $(P<0.0001)$. Of note is the high proportion of positive tumours that decreased to 0 by $10-14$ days (45.6\%). This percentage rose to $66.7 \%$ at 3 months. A comparison of 10 - to 14day biopsies with those at 3 months showed significant decreases with extended treatment with the groups of ClinR and PathR, whereas no significant difference was detected in the NR group 
(but this represents a small number of pairs). In terms of comparisons between tumours R or NR assessed either clinically or pathologically, no significant differences were detected either at pretreatment, $10-14$ days or 3 months in absolute values (data not shown).

\section{Oestrogen receptors}

There was no significant difference in ER score pretreatment, after 10-14 days and after 3 months. Neither was there a significant change with treatment with all biopsies scoring 7 or 8 throughout (data not shown).

\section{DISCUSSION}

The observation that neoadjuvant treatment with letrozole is associated with a marked reduction in the immunohistochemical expression of Ki67 and PgR confirms our previous findings (Miller et al, 2003; Anderson et al, 2004) and those of others (Ellis et al, $2001,2003)$. However, the present study extends our previous work by demonstrating that such effects are evident as early as $10-14$

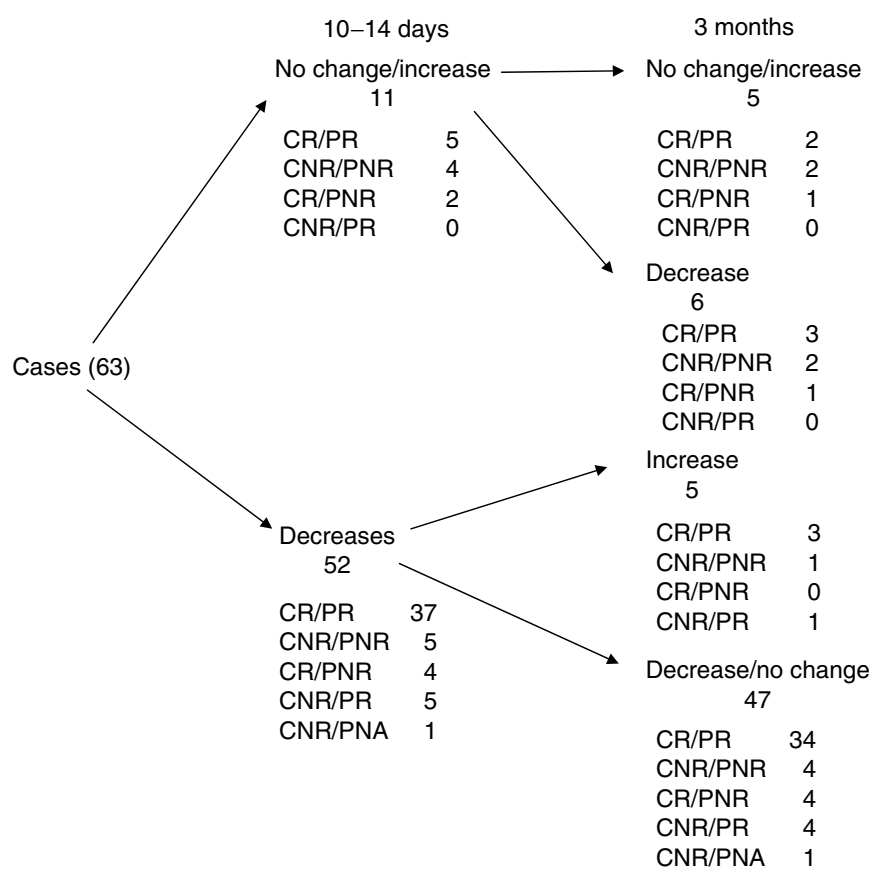

Figure 2 Flow diagram indicating number of cases grouped according to Ki67 changes at 10-14 days and 3 months and further subdivided according to final clinical/pathological response. days into treatment in over $80 \%$ of cases. Similar results have recently been presented for anastrozole (Dowsett et al, 2005a, b). The same group have also presented results from a randomised neoadjuvant trial comparing the aromatase inhibitor vorozole with tamoxifen. Ki67 levels fell within 2 weeks of treatment and remained suppressed at surgery 3 months later (Harper-Wynne et al, 2002). These effects are therefore apparent before evidence of morphological changes in tumour pathology and clinical evidence of changes in tumour volume. It was of interest in the present paper to determine whether changes in proliferation and $\mathrm{PgR}$ expression related to and/or predicted for subsequent ClinR and PathR.

In terms of assessing proliferation status with Ki-67 scores, we have analysed results in three ways: (i) comparison of tumour scores at individual time points grouped according to response status at 3 months, (ii) classifying a $>40 \%$ change in Ki-67 between different time points as evidence of a meaningful change in proliferation and (iii) comparing the number of cases in which proliferation is reduced to $<1 \%$, a value that we have regarded as a state of virtual non-proliferation. By using these multiple analyses, we hoped to derive impressions not only of group trends but effects and degree in individual cases.

Group comparisons of mean Ki-67 scores at individual study time points revealed interesting differences according to whether response was assessed clinically or pathologically. Thus, the only detectable significant differences were in groups subdivided by pathological assessment in which higher mean scores were found in non-responding tumours at both 10-14 days and 3 months. Interestingly, the same general pattern was evident when categorising individual cases according to $>40 \%$ reduction (the only significant difference was seen between PathR and PathNR at 10-14 days). The restriction of significant effects to pathological assessment probably reflects the closer association between two histological assessments rather than that between histology and tumour size. It is also worth noting that the tumour morphology after treatment will be determined by factors in addition to proliferation, such as cell loss. In this respect, although Ki67 is a primary marker of proliferation, it can also be a secondary reflection of cell death (Archer et al, 2003).

A reduction of $>40 \%$ in Ki-67 was apparent in most cases at 10-14 days, and extended treatment to 3 months was associated with only minor changes in the proportion of tumours that displayed a $>40 \%$ decrease in proliferation. This is in contrast to the results based upon the more profound criteria of a decrease to an absolute value of $<1 \%$. These results show that, remarkably, even after 10-14 days of treatment, $17 \%$ of tumours have reached this state of virtual non-proliferation, and this was irrespective of whether tumours subsequently displayed evidence of clinical or pathological response. However, the proportion of cases falling to $<1 \%$ proliferation increases further to $43 \%$ by 3 months. Interestingly, this incremental effect with time appears restricted to those tumours that had either a pathological or clinical response

Table 3 Changes in tumour PgR score with treatment

No. of patients

Changes at 10-14 days

Changes at 3 months

\begin{tabular}{ccccccccc}
\hline Increase & No change & Decrease & (Decrease to $\mathbf{0})$ & Increase & No change & Decrease & (Decrease to 0) \\
\hline I & 11 & 36 & $(18)$ & 0 & 9 & 39 & $(29)$ \\
0 & 2 & 13 & $(8)$ & 0 & 2 & 13 & $(9)$ \\
1 & 11 & 35 & $(16)$ & 0 & 9 & 38 & $(26)$ \\
0 & 2 & 13 & $(9)$ & 0 & 2 & 13 & $(11)$ \\
\hline
\end{tabular}

Pathology non-responders (15)

$\mathrm{PgR}=$ progesterone receptor. 
status at 3 months. It is clear that letrozole is capable of producing increased suppression of proliferation when used over an extended period.

The overall perspective therefore is that letrozole is capable of producing a rapid reduction in tumour proliferation that is seen in most tumours irrespective of subsequent clinical and pathological response, but that incremental effects on proliferation (as monitored by scores of $<1 \%$ ) are additionally seen in the period between 10-14 days and 3 months, and these are largely restricted to PathR or ClinR.

Whereas changes in Ki-67 levels have been revealed by group comparisons, the strength of neoadjuvant studies is that it is possible to examine differences in individual cases and classify tumours according to sequential changes in proliferation. Thus, consistent with the general trends discussed above, most tumours displayed a substantial decrease in proliferation $>40 \%$ by $10-14$ days and this was sustained at 3 months. However, it was also possible to identify (i) a small cohort that initially had decreased proliferation at 10-14 days but which largely disappeared by 3 months, (ii) tumours that failed to demonstrate a decrease in proliferation at 10-14 days but had a delayed decrease apparent at 3 months and (iii) cases that failed to display a decrease in proliferation at both 10-14 days and 3 months. It is therefore important not only to discuss relationships between clinical/ pathological response and proliferation at individual time points, but also to take into account the patterns of change in response to treatment.

Statistically significant differences were detected between PathR and PathNR in (i) group levels of Ki67 at 10-14 days and (ii) the proportion of cases decreasing in Ki67 $>40 \%$ between pretreatment and 10-14 days. However, there was a large overlap in values at $10-14$ days between PathR and PathNR, and individual tumours could display an increase, no change or a decrease in Ki67 irrespective of being PathR or PathNR. Consequently, measurements of Ki67 in individual cases do not accurately predict for subsequent pathological (or clinical) response. A similar lack of prediction between Ki67 changes and clinical response to anastrozole has been observed in the recently reported IMPACT neoadjuvant trial (Dowsett et al, 2005a) (although the short-term changes in proliferation did parallel recurrence-free survival between the three treatment groups, anastrozole, tamoxifen and Arimidex combined with tamoxifen) (Dowsett et al, 2005b). As a consequence, consideration therefore needs to be given as to why clear decreases in cellular proliferation at 10-14 days do not translate into pathological response and why conversely responding cases can show no change or even an increase in Ki67 with treatment.

It is possible that lack of correlation in some cases relates in part to imprecise measurements of proliferation or misclassification of response. In terms of immunohistochemical assessment of Ki67, we have already published data on reproducibility in breast cancer biopsies (Iqbal et al, 2002). These showed that, because of inherent heterogeneity, marked variation in Ki67 score may be observed in the same tumour without intervening treatment. However, this is restricted to occasional tumours, and the number of cases in the present study with discordance between proliferation changes and clinical/pathological response is greater than would have been expected. Furthermore, in order to reduce spurious results, we have used three different criteria for assessing changes in Ki67. In terms of the impact of assessment of clinical response, potential sources of inconsistencies have been considered elsewhere and vary according to the technology employed (Forouhi et al, 1994). In the present studies, clinical responses were based primarily upon ultrasound measurements, but they were substantiated by parallel calliper and mammographic measurements in all cases. For ease of presentation, clinical response was dichotomised and it is possible that the use of continuous variables might have been more informative. However, preliminary analyses using conti- nuous variables did not reveal better relationships between proliferation and response (data not shown). There are also limitations to the assessment of pathological response in that the pretreatment assessments (and some of the post-treatment) were performed on core biopsies, which are not guaranteed to be representative of the total tumour mass. It is also possible that assessment of ClinR/PathR at the single time point of 3 months is associated with chronological inaccuracy in that certain tumours classified as NR may go on to respond with extended treatment (Dixon et al, 2005). There is no doubt that response is not complete by 3 months and treatment up to 12 months may be associated with (a) further tumour shrinkage and (b) an increased incidence of complete ClinR.

Although methodological imprecision might be influential in some cases, it is unlikely that these totally account for the lack of association between proliferation and response. Other reasons need to be considered including the possibility that reduction in proliferation alone may not produce tumour shrinkage and cell death or apoptosis may be equally influential. Although we have not measured apoptosis in tumour samples from the present study (because assessment in core biopsies was not sufficiently reproducible), we did not find apoptosis to be predictive of response in other tumour samples from patients offered neoadjuvant endocrine therapy (Anderson et al, 2002). However, this may be because differences are small and transient; primary effects on apoptosis may also be masked by those occurring secondarily (e.g. as a result of decreased proliferation).

Another cause for a reduction in proliferation at 10-14 days not translating into subsequent tumour response could be that the effect is transient and not maintained over a sufficiently extended period to produce tumour shrinkage. However, in the present study, four of the five non-responding tumours with a reduction in Ki67 had a sustained decrease in proliferation to 3 months. A disconnect between changes in proliferation as observed at 10-14 days with subsequent clinical/pathological response could also be explained if the proliferation response was delayed. Interestingly, six of 11 tumours showed a delayed reduction in Ki67 scores; three of these were classified as PathR/ClinR.

It is worth noting that in 11 of $63(17 \%)$ cases, change in proliferation at 10-14 days failed to predict that at 3 months. Furthermore, most importantly, change in proliferation at 10-14 days failed to predict clinical response in 18 out of $63(29 \%)$ cases and pathological response in 14 out of $62(23 \%)$ tumours. Change in proliferation at 10-14 days is therefore not an accurate surrogate of clinical/pathological response at 3 months.

The other marker that showed major changes with therapy was PgR. Thus, $78 \%$ of cases displayed a reduction of at least 1 category score by 10-14 days and was maintained to 3 months. Reduction occurred irrespective of subsequent clinical/pathological response. The extent of effect may be gauged by the percentage of cases reduced to negativity ( $40 \%$ at $10-14$ days and $60 \%$ at 3 months); again the decrease to negativity was irrespective of clinical or pathological response. As PgR is a marker of signalling from the $\mathrm{ER}$, these changes are clear evidence of the anti-oestrogenic effects of letrozole and contrast with those of tamoxifen (Anderson et al, 2002; Miller et al, 2003).

Although we have not formally presented the correlations between changes in $\mathrm{PgR}$ and proliferation, there was a positive relationship between them. However, there were instances of discordance whereby at both 10-14 days and 3 months, tumours displayed either a phenotype of reduced proliferation but stable $\mathrm{PgR}$ or unchanged proliferation and reduced PgR. As reduced PgR expression is a marker of oestrogen deprivation, it is unlikely that the lack of effect on Ki67/ClinR/PathR in these cases is because of lack of aromatase inhibition. Hence, changes in PgR with treatment, although marked and occurring early, are not predictive of clinical or pathological response. It was also of interest to examine changes in PgR score in tumours that initially displayed a 
decrease in Ki67 at 10-14 days but an increase at 3 months. This phenotype could reflect adaptive changes leading to an oestrogenic stimulation or a state of hypersensitivity to oestrogen (Santen et al, 2004); however, changes in PgR in this cohort of five tumours with this phenotype revealed three cases that decreased between 10-14 days and 3 months and two cases that were negative at all time points, providing no evidence for adaptive changes or hypersensitivity to a reduced oestrogenic environment.

In conclusion, the present study has provided evidence that neoadjuvant letrozole produces marked effects on levels of Ki67 and PgR within 10-14 days. Although early changes in proliferation are less likely to occur in tumours that show no pathological response at 3 months, the effects can be seen irrespective of clinical and/or pathological response in individual cases. Ki67 scores and PgR expression are therefore of limited value as predictors of response. They do however reflect the potent anti-oestrogenic and anti-proliferative properties of the third-generation aromatase inhibitors and it has been suggested that such changes may relate to long-term outcome (Ellis et al, 2003; Dowsett et al, 2005b).

\section{ACKNOWLEDGEMENTS}

We thank Novartis for an unrestricted educational grant that was used to finance part of this work.

\section{REFERENCES}

Allred DC, Harvey JM, Berardo M, Clark GM (1998) Prognostic and predictive factors in breast cancer by immunohistochemical analysis. Mod Pathol 11: 155-168

Anderson TJ, Dixon JM, Murray J, Renshaw L, White S, Miller WR (2004) Early changes in tumour Ki67 expression differentiate for pathological (but not clinical) response in breast cancers treated neoadjuvantly with letrozole. Breast Cancer Res Treat 88(Suppl 1): S50

Anderson TJ, Dixon JM, Stuart M, Sahmoud T, Miller WR (2002) Effect of neoadjuvant treatment with anastrozole on tumour histology in postmenopausal women with large operable breast cancer. Br J Cancer 87: $334-338$

Archer CD, Parton M, Smith IE, Ellis PA, Salter J, Ashley S, Gui G, Sacks N, Ebbs SR, Allum W, Nasiri N, Dowsett M (2003) Early changes in apoptosis and proliferation following primary chemotherapy for breast cancer. Br J Cancer 89: 1035 - 1041

Dixon JM (2001) Neoadjuvant endocrine therapy. In Aromatase Inhibition and Breast Cancer, Miller WR, Santen RJ (eds). pp 103-116. UK: Pub Marcel Dekker

Dixon JM, Love CD, Bellamy CO, Cameron DA, Leonard RC, Smith H, Miller WR (2001) Letrozole as primary medical therapy for locally advanced and large operable breast cancer. Breast Cancer Res Treat 66(3): $191-199$

Dixon JM, Renshaw L, Murray J, Macaskill EJ, Young O, Miller WR (2005) Surgical issues surrounding use of aromatase inhibitors. J Steroid Biochem Mol Biol 95: 97-103

Dowsett M, Ebbs SR, Dixon JM, Skene A, Griffith C, Boeddinghaus I, Salter J, Detre S, Hills M, Ashley S, Francis S, Walsh G, Smith IE (2005a) Biomarker changes during neoadjuvant anastrozole, tamoxifen, or the combination: influence of hormonal status and HER-2 in breast cancer a study from the IMPACT trialists. J Clin Oncol 23(11): 2477-2492

Dowsett M, Smith IE, Ebbs SR, Dixon JM, Skene A, Griffith C, Boeddinghaus I, Salter J, Detre S, Hills M, Ashley S, Francis S, Walsh G, on behalf of the IMPACT Trialists (2005b) Short-term changes in Ki67 during neoadjuvant treatment of primary breast cancer with anastrozole or tamoxifen alone or combined correlate with recurrence-free survival. Clin Cancer Res 11(Suppl 1): 951s-958s

Eiermann W, Paepke S, Appfelstaedt J, Llombart-Cussac A, Eremin J, Vinholes J, Mauriac L, Ellis M, Lassus M, Chaudri-Ross HA, Dugan M, Borgs M, Letrozole Neo-Adjuvant Breast Cancer Study Group (2001) Preoperative treatment of postmenopausal breast cancer patients with letrozole: a randomized double-blind multicenter study. Ann Oncol 12(11): $1527-1532$

Ellis MJ, Coop A, Singh B, Mauriac L, Llombert-Cussac A, Janicke F, Miller WR, Evans DB, Dugan M, Brady C, Quebe-Fehling E, Borgs M (2001) Letrozole is more effective neoadjuvant endocrine therapy than tamoxifen for ErbB-1- and/or ErbB-2-positive, estrogen receptor-positive primary breast cancer: evidence from a phase III randomized trial. J Clin Oncol 19(18): $3808-3816$
Ellis MJ, Coop A, Singh B, Tao Y, Llombart-Cussac A, Janicke F, Mauriac L, Quebe-Fehling E, Chaudri-Ross HA, Evans DB, Miller WR (2003) Letrozole inhibits tumor proliferation more effectively than tamoxifen independent of HER1/2 expression status. Cancer Res 63(19): 6523-6531

Ellis PA, Smith IE, Detre S, Burton SA, Salter J, A'Hern R, Walsh G, Johnston SR, Dowsett M (1998) Reduced apoptosis and proliferation and increased $\mathrm{Bcl}-2$ in residual breast cancer following preoperative chemotherapy. Breast Cancer Res Treat 48(2): 107-116

Forouhi P, Walsh JS, Anderson TJ, Chetty U (1994) Ultrasonography as a method of measuring breast tumour size and monitoring response to primary systemic treatment. Br J Surg 81(2): 223-225

Going JJ (1994) Efficiently estimated histologic cell counts. Hum Pathol 4: $333-336$

Goss PE, Ingle JN, Martino S, Robert NJ, Muss HB, Piccart MJ, Castiglione $M$, Tu D, Shepherd LE, Pritchard KI, Livingston RB, Davidson NE, Norton L, Perez EA, Abrams JS, Therasse P, Palmer MJ, Pater JL (2003) A randomized trial of letrozole in postmenopausal women after five years of tamoxifen therapy for early-stage breast cancer. N Engl J Med 349(19): $1793-1802$

Harper-Wynne CL, Sacks NP, Shenton K, MacNeill FA, Sauven P, Laidlaw IJ, Rayter Z, Miall S, Howes A, Salter J, Hills MJ, Lowe FM, A'Hern R, Nasiri N, Doody D, Iqbal J, Dowsett M (2002) Comparison of the systemic and intratumoural effects of tamoxifen and the aromatase inhibitor vorozole in postmenopausal patients with primary breast cancer. J Clin Oncol 20(4): 1026-1035

Iqbal S, Anderson TJ, Marson LP, Prescott RJ, Dixon JM, Miller WR (2002) MIB-1 assessments in breast cancers. Breast 11(3): 252-256

Miller WR, Dixon JM, Macfarlane L, Cameron D, Anderson TJ (2003) Pathological features of breast cancer response following neoadjuvant treatment with either letrozole or tamoxifen. Eur J Cancer 39(4): $462-468$

Mouridsen H, Gershanovich M, Sun Y, Perez-Carrion R, Boni C, Monnier A, Apffelstaedt J, Smith R, Sleeboom HP, Janicke F, Pluzanska A, Dank M, Becquart D, Bapsy PP, Salminen E, Snyder R, Lassus M, Verbeek JA, Staffler B, Chaudri-Ross HA, Dugan M (2001) Superior efficacy of letrozole $v s$ tamoxifen as first-line therapy for postmenopausal women with advanced breast cancer: results of a phase III study of the International Letrozole Breast Cancer Group. J Clin Oncol 19: 2596-2606

Rose C, Vtoraya O, Pluzanska A, Davidson N, Gershanovich M, Thomas R, Johnson S, Caicedo JJ, Gervasio H, Manikhas G, Ben Ayed F, BurdetteRadoux S, Chaudri-Ross HA, Lang R (2003) An open randomised trial of second-line endocrine therapy in advanced breast cancer: comparison of the aromatase inhibitors letrozole and anastrozole. Eur J Cancer 39(16): $2318-2327$

Santen RJ, Song RX, Zhang Z, Yue W, Kumar R (2004) Adaptive hypersensitivity to estrogen: mechanism for sequential responses to hormonal therapy in breast cancer. Clin Cancer Res 10(1 Part 2): $337 \mathrm{~S}-345 \mathrm{~S}$ 